I.A. Karimi and Rajagopalan Srinivasan (Editors), Proceedings of the 11th International Symposium on Process Systems Engineering, 15-19 July 2012, Singapore.

(C) 2012 Elsevier B.V. All rights reserved.

\title{
A Novel Global Optimization Approach to the Multiperiod Blending Problem
}

\author{
Scott P. Kolodziej, ${ }^{\mathrm{a}}$ Ignacio E. Grossmann ${ }^{\mathrm{a}}$ \\ ${ }^{a}$ Department of Chemical Engineering, Carnegie Mellon University, 5000 Forbes Ave, \\ Pittsburgh PA, 15213, USA
}

\begin{abstract}
In this paper, we introduce a generalized multiperiod version of the pooling problem to represent time varying blending systems, and also propose novel approaches to solve these problems to global optimality. The primary difficulties in solving this optimization problem are the presence of bilinear terms inherent in blending operations, as well as binary decision variables required to impose the operational constraints over multiple time periods. A general nonconvex MINLP formulation is presented that is used to globally optimize small systems, but quickly becomes intractable as problem size increases. A novel approximation of specified precision for the nonconvex bilinear terms is developed (a radix-based discretization scheme), with which the problem can be reformulated as an MILP. Solving this new formulation requires much less computational time than when the MINLP model is solved directly with a global optimization solver such as BARON. This then allows for the global optimization of larger blending systems. A comparison of the two formulations is presented, along with detailed computational results of each approach.
\end{abstract}

Keywords: global optimization, pooling problem, MINLP, discretization, radix-based

\section{Introduction}

The efficient blending of liquid fuels to meet both technical and environmental specifications has been a growing research area in recent years as stricter regulations and smaller profit margins drive the need for the globally optimal blending scheme (Misener \& Floudas, 2009). Specifically in refineries, the blending of different distilled fractions to meet specifications - without waste - is of great importance. Research up to this point has focused on what has become known as the pooling problem, posed by Haverly in 1978. In short, the problem is as follows: Multiple liquid streams with various properties (called qualities) are fed from supply tanks into blending tanks where they are assumed to be perfectly mixed in some proportion to meet a set of specifications, and are then fed into demand tanks. The goal is then to select the flows that minimize the overall cost of the blending process. The traditional pooling problem is assumed to operate at steady state, and thus inventory and other dynamics are neglected. In practice, however, supply and demand vary with time, and therefore the inventory in each tank varies as well. This gives rise to a multiperiod blending problem, which has received very little attention in the literature. Therefore, unlike the pooling problem, the supply and demand flows in the multiperiod problem are specified as a function of time. Additionally, mass balances over each blending tank must allow for accumulation in the form of inventories, as flow into a tank need not equal the flow leaving the tank. Lastly, a special operational constraint is imposed: flow cannot both enter and exit a blending tank in the same time period. This avoids the dynamic change of concentration associated with filling and draining a tank at the same time. The 
multiperiod blending problem allows for a more accurate modeling of a blending system that varies over time, as is the case in gasoline and crude oil blending in refineries.

\section{Multiperiod Blending Problem}

\subsection{Problem Description}

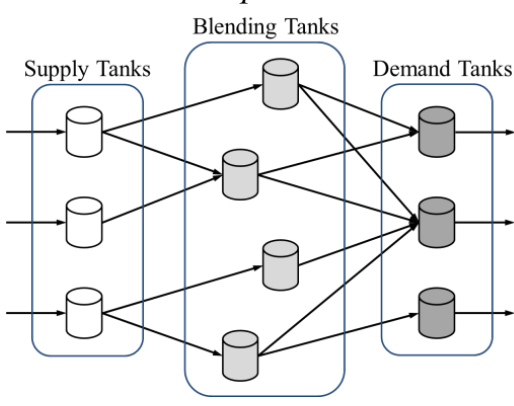

Figure 1: The pooling problem

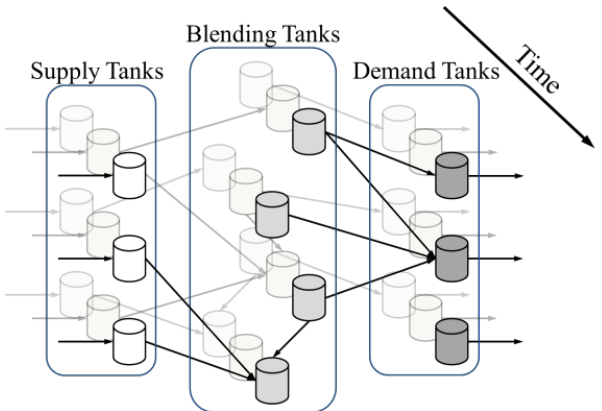

Figure 2: The multiperiod blending problem

The multiperiod blending problem can be stated as follows. A network of supply, blending, and demand tanks is given that operates over a time horizon defined by a set of time periods. At time $t=0$, an initial inventory is specified for each tank, as well as initial values for the qualities. Given the specified network topology, the optimal flows between the tanks in each time period must be determined, as well as the corresponding inventory levels, which are carried over from one period to another. It is important to note that each time period is not independent of the others due to the coupling created by the inventories. For example, flow may be diverted to a tank for temporary storage to be used in a later period, or there may be no direct path from a source tank to a demand tank. Thus, the optimization must be performed simultaneously over all time periods.

In detailed terms, incoming supply flows $\left(F_{s, t}\right)$ enter supply tanks each time period, and demand flows $\left(F_{d, t}\right)$ are withdrawn from the demand tanks each time period. The supply flows to a given tank are assumed for simplicity to have the same composition over all time $\left(C_{q, s}\right)$ but can vary in amount (hence the subscript $t$ in $\left.F_{s, t}\right)$. Likewise, the concentration of flows leaving the demand tanks must be within specified bounds $\left(C_{q, d}^{L}\right.$ and $C_{q, d}^{U}$ ), but the flows can also vary in amount (hence the subscript $t$ in $F_{d, t}$ ). Bounds on inventories are also given for each tank $\left(I_{n}{ }_{n}\right.$ and $\left.I_{n}{ }_{n}\right)$ and for each flow between each tank $\left(F_{n \rightarrow n}^{L}\right.$, usually zero, and $\left.F_{n \rightarrow n}^{U}\right)$. Lastly, costs for the supply flows $\left(\beta_{s}\right)$, demand flows $\left(\beta_{d}\right)$, and fixed and variable costs for flows within the network $(\alpha$ and $\beta$, respectively) are taken into account with the goal of maximizing the profit (or minimizing the costs) of the multiperiod network operation to most efficiently mix the fuels to meet demand specifications. Because of the operational constraint that flow cannot both enter and exit a blending tank in the same time period, as well as to represent the fixed costs, binary decision variables $y_{n \rightarrow n, t}$ must be introduced into the problem such that $y_{n \rightarrow n^{\prime}, t}=1$ when any flow exists between tank $n$ and tank $n$ ' in time period $t$, and $y_{n \rightarrow n^{\prime}, t}=0$ otherwise.

\subsection{MINLP Formulation}

Given a network topology and all associated values described above, the schedule for blending the fuels is sought that maximizes total profit. As stated earlier, the multiperiod blending problem naturally involves binary variables $y_{n \rightarrow n}{ }^{\prime}$ for all the streams in each time period, and bilinearities for the mass balance constraints involving 
mixing. This leads to the following nonconvex mixed-integer nonlinear programming (MINLP) model:

$$
\begin{aligned}
& \operatorname{Max} \sum_{t \in T}\left[\sum_{b \in B} \sum_{d \in D} \beta_{d} F_{b \rightarrow d, t}-\sum_{s \in S} \sum_{b \in B} \beta_{S} F_{s \rightarrow b, t}-\sum_{n \in N} \sum_{n^{\prime} \in N}\left(\alpha_{n \rightarrow n^{\prime}} y_{n \rightarrow n^{\prime}, t}+\beta_{n \rightarrow n^{\prime}} F_{n \rightarrow n^{\prime}, t}\right)\right] \\
& \text { Subject To: } \\
& \begin{array}{ll}
F_{n \rightarrow n^{\prime}, t} \leq F_{n \rightarrow n^{\prime}}^{U} y_{n \rightarrow n^{\prime}, t} & \forall n, n^{\prime} \in N ; t \in T \\
F_{n \rightarrow n^{\prime}, t} \geq F_{n \rightarrow n^{\prime}}^{L} y_{n \rightarrow n^{\prime}, t} & \forall n, n^{\prime} \in N ; t \in T \\
C_{q, b, t} \leq C_{q, d}^{U}+M\left(1-y_{b \rightarrow d, t-1}\right) & \forall q \in Q ; d \in D ; t \in T \\
C_{q, b, t} \geq C_{q, d}^{L}+M\left(1-y_{b \rightarrow d, t-1}\right) & \forall q \in Q ; d \in D ; t \in T \\
C_{q, b, t} \leq C_{q, d}^{U}+M\left(1-y_{s \rightarrow d, t-1}\right) & \forall q \in Q ; s \in S ; t \in T \\
C_{q, b, t} \geq C_{q, d}^{L}+M\left(1-y_{s \rightarrow d, t-1}\right) & \forall q \in Q ; s \in S ; t \in T \\
I_{s, t}=I_{s, t-1}+F_{s, t}-\sum_{n \in N} F_{s \rightarrow n, t} & \forall s \in S ; t \in T \\
I_{b, t}=I_{b, t-1}+\sum_{n \in N} F_{n \rightarrow b, t}-\sum_{n \in N} F_{b \rightarrow n, t} & \forall b \in B ; t \in T \\
I_{d, t}=I_{d, t-1}+\sum_{n \in N} F_{n \rightarrow d, t}-F_{d, t} & \forall d \in D ; t \in T \\
I_{b, t} C_{q, b, t}=I_{b, t-1} C_{q, b, t-1}+\sum_{s \in S} F_{s \rightarrow b, t} C_{q, s} & \\
\quad+\sum_{b^{\prime} \in B} F_{b^{\prime} \rightarrow b, t} C_{q, b^{\prime}, t-1} & \forall q \in Q ; b \in B ; t \in T \\
\quad-\sum_{n \in N} F_{b \rightarrow n, t} C_{q, b, t-1} & \\
y_{n \rightarrow b, t}+y_{b \rightarrow n^{\prime}, t} \leq 1 & \forall b \in B ; n, n^{\prime} \in N \\
I_{n}^{L} \leq I_{n, t} \leq I_{n}^{U} & \forall n \in N ; t \in T \\
y_{n \rightarrow n^{\prime}, t} \in\{0,1\} & \forall n, n^{\prime} \in N ; t \in T \\
F_{n \rightarrow n^{\prime}, t} \geq 0 ; \quad I_{n, t} \geq 0 ; & \\
&
\end{array}
\end{aligned}
$$

To solve the multiperiod blending problem, the MINLP formulation must be solved to global optimality due to the bilinear constraints in (5).

\section{Radix-based Discretization}

Because the primary difficulty is the presence of bilinear terms (the only nonlinearity), we propose an approximation for the bilinear term $\mathrm{u}=\mathrm{F} \cdot \mathrm{C}$ by introducing a novel radixbased discretization (RBD) of $\mathrm{C}$. This approach was proposed in the solution of water network problems by Teles, Castro, \& Matos (2012). The approximation shown below, which can be derived using disjunctive programming and exact linearizations, replaces the bilinear terms F.C in equation 5 of the MINLP formulation (I.C is treated similarly):

$$
\begin{aligned}
& u=\sum_{k=p}^{P} \sum_{j=0}^{9} 10^{k} \cdot j \cdot \hat{F}_{j, k} \\
& C=\sum_{k=p}^{P} \sum_{j=0}^{9} 10^{k} \cdot j \cdot z_{j, k} \\
& \hat{F}_{j, k} \leq F^{U} \cdot z_{j, k} \\
& \sum_{j=0}^{9} \hat{F}_{j, k}=F
\end{aligned}
$$




$$
\begin{array}{ll}
\sum_{j=0}^{9} z_{j, k}=1 & \forall k \in\{p, \ldots, P\} \\
z_{j, k} \in\{0,1\} ; \quad \hat{F}_{j, k} \geq 0 & \forall k \in\{p, \ldots, P\}, j \in\{0, \ldots, 9\}
\end{array}
$$

This set of constraints essentially represents $\mathrm{C}$ as a discrete value based on a given radix (in this case 10) to arbitrary precision (determined by $\mathrm{p}$, the smallest power of 10 allowed, and $\mathrm{P}$, the largest power of 10 allowed). Other radices (or bases) can also be used. The accuracy of the solution depends on the precision of this approximation. However, a global or near global solution can be found by fixing the binary variables to the values found by the approximation and resolving the problem with a global or local NLP solver. Also, we should note that to ensure feasible solutions that the tolerances for the satisfaction of constraints need to be adjusted accordingly.

While the proposed reformulation introduces significantly more constraints and variables, it changes the class of the problem from an MINLP to a mixed-integer linear programming problem (MILP). This class of optimization problem can generally be solved more efficiently, especially using highly refined commercial solvers such as IBM ILOG's CPLEX Optimizer (Int, 2009) and Gurobi Optimization's Gurobi Optimizer (Gur, 2011). These solvers can also take advantage of multiple computing threads, a further advantage over current MINLP solvers.

\section{Computational Results}

The nonconvex MINLP model was implemented in the modeling language GAMS (Brook et al, 1988) and solved using BARON 9.3.1 (Sahinidis, 1996) to guarantee a global optimum. For comparison, the discretized MILP formulation was also implemented in GAMS and solved using Gurobi 4.5.1. Using this new radix-based discretization approach at a discretization of $\mathrm{p}=-3$ and $\mathrm{P}=0\left(10^{-3}\right.$ to $\left.10^{0}\right)$, we can solve previously intractable problems (requiring BARON more than 6 hours of computational time to close the optimality gap to $0.1 \%$ ) in minutes. The results of this approach and the MINLP formulation solved by BARON are shown in Table 1. All computations were performed on an Intel Core i7 processor at $2.93 \mathrm{GHz}$. The Gurobi results used eight threads simultaneously, while BARON could only utilize one thread.

For an 8 tank, 3 time period problem with 2 qualities selected from the test problems, the optimal schedule is shown in Figure 3 and detailed results in Table 2.

Table 1: Computational results of MINLP (BARON) and MILP (Gurobi) formulations for five test multiperiod blending problems

\begin{tabular}{|l|r|r|r|r|r|}
\hline Tanks & 6 & 8 & 8 & 8 & 8 \\
\hline Time Periods & 3 & 3 & 3 & 4 & 4 \\
\hline MINLP CPU Time (s) & 17.7 & $>21600.0$ & $>21600.0$ & $>21600.0$ & $>21600.0$ \\
\hline RBD CPU Time (s) & 3.0 & 663.6 & 389.7 & 845.9 & 409.7 \\
\hline CPU Time Speedup & $5.9 \mathrm{x}$ & $>32.5 \mathrm{x}$ & $>55.4 \mathrm{x}$ & $>25.5 \mathrm{x}$ & $>52.7 \mathrm{x}$ \\
\hline
\end{tabular}

Table 2: Computational results for 8 tank, 3 time period, 2 quality problem

\begin{tabular}{|l|r|r|r|r|r|}
\hline & CPU Time (s) & Objective & Constraints & $\begin{array}{c}\text { Continuous } \\
\text { Variables }\end{array}$ & $\begin{array}{c}\text { Binary } \\
\text { Variables }\end{array}$ \\
\hline MINLP & $>21600.0$ & $* 13.4517$ & 628 & 136 & 87 \\
\hline RBD (MILP) & 389.7 & 13.5268 & 7092 & 4632 & 855 \\
\hline NLP Post-Solve & 0.2 & 13.5268 & 447 & 327 & 0 \\
\hline
\end{tabular}

*Best lower bound 
While the discretization approach greatly increases the problem size, the time required to solve the problem is decreased significantly. Additionally, after solving the discretized MILP, the binary variables present in the original problem were fixed and the problem was solved again with BARON acting as an NLP solver (see Table 2).
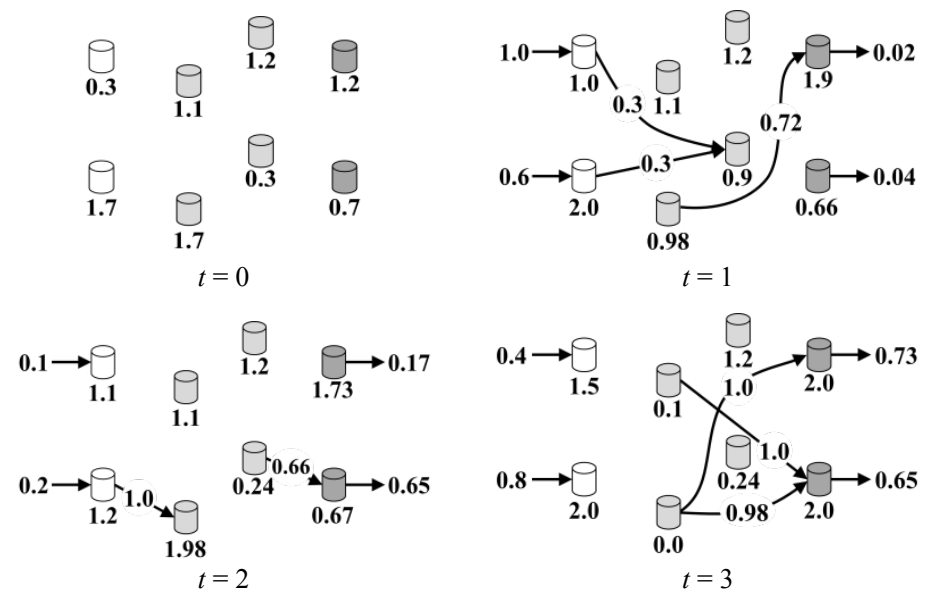

Figure 3: Optimal solution for 8 tank, 3 time period, 2 quality problem

\section{Conclusion}

We have addressed in this paper the multiperiod blending problem for which a nonconvex MINLP formulation was proposed. It was shown that the radix-based discretization approach has allowed the solution of larger multiperiod blending problems, with up to 8 tanks and 4 time periods, in a significantly shorter amount of time. However, as problem size increases, the discretized approach may become intractable. Thus, for future investigation, decomposition approaches will likely be required for the solution of very large systems.

\section{Acknowledgements}

We would like to thank ExxonMobil Corporation for supporting this research.

\section{References}

Brook, A.; Kendrick, D.; and Meeraus, A. 1988. GAMS, a user's guide. SIGNUM Newsletter 23:10-11.

Gurobi Optimization. 2011. Gurobi Optimizer Reference Manual Version 4.5.

Haverly, C. A. 1978. Studies of the behavior of recursion for the pooling problem. SIGMAP Bulletin 19-28.

International Business Machines Corporation. 2009. IBM ILOG CPLEX V12.1: User's Manual for CPLEX.

Misener, R., and Floudas, C. A. 2009. Advances for the pooling problem: Modeling, global optimization, and computational studies. Applied and Computational Mathematics 8(1):3 22.

Sahinidis, N. V. 1996. BARON: A general purpose global optimization software package. Journal of Global Optimization 8:201-205.

Teles, J. P.; Castro, P. M.; and Matos, H. A. 2012. New global optimization approach for the optimal design of industrial water networks. Submitted for publication. 